\title{
Vitiligo: A new side effect of everolimus therapy for metastatic renal cell
}

\section{carcinoma}

Sara Cacciapuoti ${ }^{1}$, Anna Masarà ${ }^{1}$, Maria Mariano ${ }^{2}$, Norma Cameli ${ }^{2}$, and Gabriella Fabbrocini ${ }^{1}$

1. Section of Dermatology, Department of Clinical Medicine and Surgery, University of Naples Federico II, Via Pansini 5, 80131, Napoli, Italy

2. Istituto Dermatologico San Gallicano-IRCCS-Roma, Italy

\section{CASE STUDY}

Please cite this paper as: Cacciapuoti S, Masarà A, Mariano M, Cameli N, Fabbrocini G. Vitiligo: A new side effect of everolimus therapy for metastatic renal cell carcinoma. AMJ 2017;10(10):898-900.

https://doi.org/10.21767/AMJ.2017.3131

\section{Corresponding Author:}

Sara Cacciapuoti

Section of Dermatology

Department of Clinical Medicine and Surgery

University of Naples Federico II

Via Pansini 5, 80131, Napoli, Italy

E-mail: sara.cacciapuoti@libero.it

\section{ABSTRACT}

Patients with metastatic renal cell carcinoma ( $\mathrm{mRCC}$ ) have always had a poor prognosis. Recently new targeted drugs were developed. A 73-year-old female patient affected from mRCC was assessed at our Department. She underwent before a multitargeted tyrosine kinase inhibitor (TKI) therapy, but she developed progressive liver metastases so she was treated after with everolimus, an allosteric inhibitor of the mammalian target of rapamycin (mTOR), but started developing depigmented lesions over the neck. Vitiligo is a common cutaneous disorder and drug-induced vitiligo is reported. Our case can suggest a new type of drug-induced vitiligo, caused by a melanocyte-specific mechanism of toxicity.

\section{Key Words}

Everolimus, mTOR, metastatic renal cell carcinoma, vitiligo

\section{Implications for Practice:}

\section{What is known about this subject?}

Vitiligo is a cutaneous disorder whose pathogenesis has been related to a combination of mechanisms leading to melanocyte impairment and loss. Drug-induced vitiligo is reported.

\section{What new information is offered in this case study?}

Our case can suggest a new type of drug-induced vitiligo, caused by a melanocyte-specific mechanism of toxicity not reported, until now, in literature.

3. What are the implications for research, policy, or practice?

The doctor must not be unprepared keeping in mind the possible onset of this specific new side effect during everolimus therapy.

\section{Background}

Patients with metastatic renal cell carcinoma (mRCC) have always had a poor prognosis due to the inefficacy of systemic chemotherapy and immunotherapy to treat these tumours. Fortunately, the management of patients with mRCC has changed dramatically over the past few years. Before 2005, medical therapies for mRCC were limited to cytokine therapies, which are very toxic and benefit only a small percentage of patients. Recently, new targeted drugs were proposed, including Tyrosine kinase inhibitors, checkpoint inhibitors, and combinations of these new agents that can improve outcomes for patients. ${ }^{1}$ Among these, small-molecule multitargeted tyrosine kinase inhibitors (TKIs) such as sorafenib, sunitinib and mammalian target of rapamycin (mTOR) inhibitors such as temsirolimus and everolimus seem to be able to change the natural history of this tumor. ${ }^{2}$ Toxicities during the early course of treatment are majorly caused from mTOR inhibitors and include hyperglycaemia, hypophosphatemia, weakness, anorexia, diarrhoea and skin reactions such as leukocytoclastic vasculitis. ${ }^{3}$ As recently introduced drugs, it 
is necessary to keep in mind the possible occurrence of new side effects, not yet reported.

\section{Case details}

A 73-year-old female patient affected from $\mathrm{mRCC}$ was assessed in June 2015 at our Department. In April 2012 she underwent a multitargeted tyrosine kinase inhibitor (TKI) therapy (Sunitinib), but she developed progressive liver metastases after 8 weeks of treatment. From September 2013, she was treated with everolimus, an allosteric inhibitor of the mammalian target of rapamycin (mTOR). In our patient none of the classic toxicities developed, but three weeks after beginning everolimus therapy she started developing depigmented lesions over the neck and the décolleté (Figure 1). These lesions rapidly evolved and became confluent. At our observation she showed an anagen distrophic defluvium (Figure 2), caused by previous Sunitinib therapy, and well-defined depigmented patches involving the neck and décolleté with a little hyperpigmentation on the border. No other area of the body showed similar clinical lesions. There was no personal or family history of any autoimmune disorder and in particular, of vitiligo. Autoimmune markers were also negative in this patient. We performed a punch biopsy from a lesion of the left neck, in order to define diagnosis. Histopathologic findings showed a sparse superficial perivascular infiltrate of lymphocytes and the absence of melanocytes and melanin within the epidermis.

\section{Discussion}

Vitiligo is a common cutaneous disorder, clinically characterized by white macules on the skin that can be localized or generalized. Hypopigmentation can be caused by a defect in melanin synthesis or transfer; or by a partial or complete absence of melanocytes from the epidermis. The pathogenesis of vitiligo has been related to a combination of mechanisms leading to melanocyte impairment and loss, including stress, drugs, infections and autoimmunity. Vitiligo has been described in association with several drugs as well as with viral infections. Druginduced vitiligo is frequently reported. ${ }^{4-7}$

Some implicated drugs are anticonvulsants, antimalarials, antiparkinson's drugs and biological drugs such as infliximab, imiqimod and imatinib. The suggested etiopathogenesis behind drug-induced vitiligo is: (1) activation of cytotoxic $T$ cells against melanocyte antigens (2) damage to sympathetic nerves that are connected by chemical synapses to melanocytes with a resultant functional disturbance, and (3) a direct cytotoxic nature of the drug on melanocytes (apoptosis). Most of the mechanisms suggested are hypothetical with no direct or scientific evidence to establish the exact role of the implicated drug. Moreover several studies seem to be controversial. For example, although some studies suggest that chimerical monoclonal antibodies that inhibit proinflammatory activity of tumour-necrosis factor alpha (TNF $\alpha$ ), such as infliximab might be an effective treatment for vitiligo, ${ }^{8,9}$ but many cases reported in the literature indicate that vitiligo might also be induced by treatment with infliximab. ${ }^{10-12}$

Everolimus is a mTOR inhibitor, which was approved in March 2009 by the US FDA for treatment of targetedtherapy refractory metastatic renal cell cancer. There are no studies reporting everolimus-induced vitiligo. In our patient direct involvement of everolimus molecule seems to be very likely upon chronological data. As Pópuloù et al. reported mTOR controls proliferation and cell growth of different cells and it is over-expressed in many malignant melanomas. $^{13}$ We know also that melanocytes are responsible for melanin production and transport to surrounding keratinocytes through the junction between dendrites of melanocytes and membrane of keratinocytes, so dendrites play critical roles of melanosomal transfer in melanocytes. $^{14,15}$ The damage of dendrites, therefore, may also affect melanin transport leading to vitiligo. Like Jerry Wan et al. showed, oxidative stress reduces dendrites and normally $\alpha-\mathrm{MSH}$ protects against loss of dendrites, oxidative stress-induced, via activation of mTORC1 pathway. This may support, in our opinion, a possible causal association between onset of vitiligo and everolimus on therapy. Although the mechanism involved in this type of druginduced vitiligo isn't completely clear, we can suppose that one of the pathogenetic mechanisms is that everolimus can inhibit melanogenesis, by inhibiting the protective mTORC1 pathway against oxidative stress. ${ }^{16}$ Our case can suggest a new possible type of drug melanocyte-specific toxicity.

\section{Conclusion}

It is necessary to continue to study and keep in mind the possible occurrence of this new drug-induced side effect, not yet reported in literature, but observed in our patient.

\section{References}

1. Posadas EM, Limvorasak S, Figlin RA. Targeted therapies for renal cell carcinoma. Nat Rev Nephrol. 2017;13(8):496-511.

2. Dumont FJ, Su Q. Mechanism of action of the immunosuppressant rapamycin. Life Sci. 1996;58(5):373-95. 
3. Yee KW, Zeng Z, Konopleva M, et al. Phase I/II study of the mammalian target of rapamycin inhibitor everolimus (RAD001) in patients with relapsed or refractory hematologic malignancies. Clin Cancer Res. 2006;12(17):5165-73.

4. Gowda S, Tillman DK, Fitzpatrick JE, et al. Imiquimodinduced vitiligo after treatment of nodular basal cell carcinoma. J Cutan Pathol. 2009;36(8):878-881.

5. Sriprakash K, Godbolt A. Vitiligo-like depigmentation induced by imiquimod treatment of superficial basal cell carcinoma. Australas J Dermatol. 2009;50:211-213.

6. Cerchione C, Fabbricini R, Pane F, et al. Vitiligo-like lesions in an adult patient treated with imatinib mesylate. Leuk Res. 2009;33(8):e104-5.

7. Curzytek K, Pietowska J, Spiewak R. Vitiligo induced by drugs: a meta-analysis of reported cases. Allergy 2007;62(suppl 83:XXVI Congress of the European Academy of Allergology and Clinical Immunology):447.

8. Simón JA, Burgos-Vargas R. Vitiligo improvement in a patient with ankylosing spondylitis treated with infliximab. Dermatology. 2008;216(3):234-5.

9. Lv $Y, L i ~ Q, ~ W a n g ~ L$, et al. Use of anti-tumor necrosis factor agents: a possible therapy for vitiligo. Med Hypotheses. 2009;72(5):546-7.

10. Ramírez-Hernández M, Marras C, Martínez-Escribano JA. Infliximab-induced vitiligo. Dermatology. 2005;210(1):79-80.

11. Ismail WA, Al-Enzy SA, Alsurayei SA, et al. Vitiligo in a patient receiving infliximab for refractory ulcerative colitis. Arab J Gastroenterol. 2011;12(2):109-11.

12. AlGhamdi KM, Khurrum H, Rikabi A. Worsening of vitiligo and onset of new psoriasiform dermatitis following treatment with infliximab. J Cutan Med Surg. 2011;15(5):280-4.

13. Pópulo H, Lopes JM, Soares P. The mTOR Signalling Pathway in Human Cancer. Int J Mol Sci. 2012;13(2):1886-1918.

14. Scott G. Rac and rho: the story behind melanocyte dendrite formation. Pigment Cell Res. 2002;15:322-30.

15. Scott G, Leopardi S, Printup S, et al. Filopodia are conduits for melanosome transfer to keratinocytes. J Cell Sci. 2002;115:1441-51.

16. Wan J, Lin F, Zhang W, et al. Novel approaches to vitiligo treatment via modulation of mTOR and NF-KB pathways in human skin melanocytes. Int J Biol Sci. 2017;13(3):391-400.

\section{PEER REVIEW}

Not commissioned. Externally peer reviewed.

\section{CONFLICTS OF INTEREST}

The authors declare that they have no competing interests.

\section{FUNDING}

None

\section{PATIENT CONSENT}

The authors: Cacciapuoti S, Masarà A, Mariano M, Cameli $N$, Fabbrocini $G$, declare that:

1. They have obtained written, informed consent for the publication of the details relating to the patient in this report.

2. All possible steps have been taken to safeguard the identity of the patient.

3. This submission is compliant with the requirements of local research ethics committees.

\section{Figures}

Figure 1: Everolimus induced depigmented lesions over the neck and the décolleté in a 73-year-old female patient affected from metastatic renal carcinoma

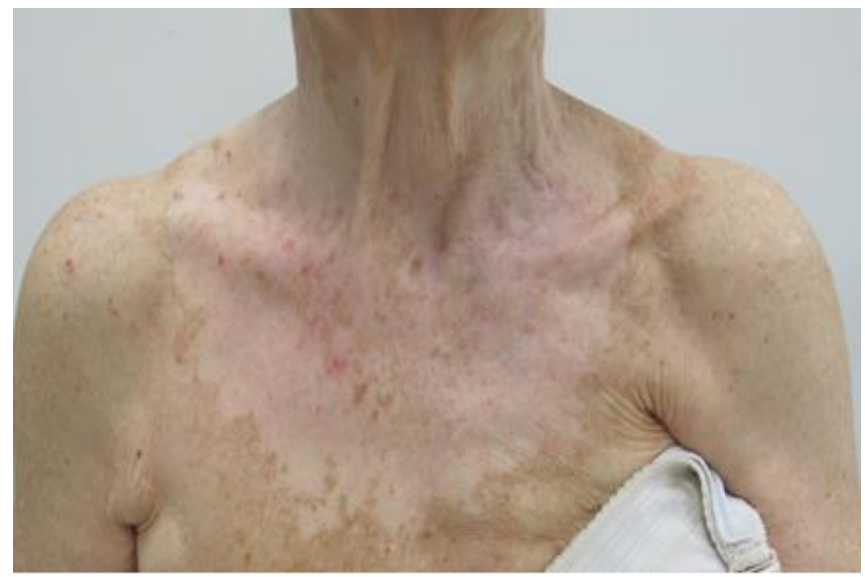

Figure 2: Anagen distrophic defluvium and well-defined depigmented patches involving the neck

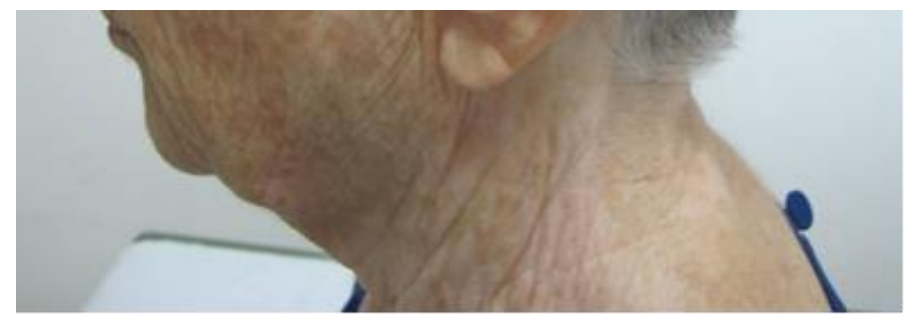

